\title{
Photometric Micro Measuring Sensor for Real-Time Water Analysis
}

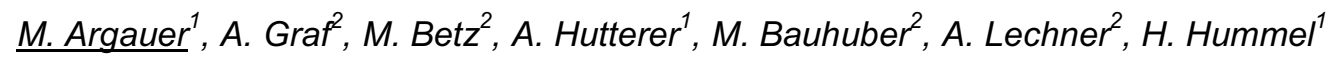 \\ ${ }_{1}^{1}$ Hochschule Regensburg, Kompetenzzentrum Sensorik, Seybothstr. 2, 93053, Germany, \\ Monika.Argauer@hs-regensburg.de, \\ ${ }^{2}$ Hochschule Regensburg, Kompetenzzentrum Nanochem, Seybothstr. 2, 93053, Germany
}

\begin{abstract}
:
The measuring sensor is a micro-fluidic cell for real-time water analysis. It can be optimized for different application fields depending on their expected contamination grade in the ppm (parts per million) to $\mathrm{ppb}$ (parts per billion) area. The cell finds its implementation especially in agricultural and environmental monitoring. It was integrated in a device called Quicklyzer for continuous measuring of soil water. The detection combines the principle of absorption and fluorescence spectroscopy. So different substances like nitrate, nitrite and humic acid can be measured in one device. The specialty is the nanotechnological liquid core waveguide which is formed with semiconductor structuring techniques. It has a surface with best homogeneity, with an optical wall roughness of $10 \mathrm{~nm}+/-2 \mathrm{~nm}$. Samples can be measured over long distances on a small size, e.g. a cell with a length of $2.8 \mathrm{~m}$ integrated on 6" silicon substrate. The channel must be coated with a total internal reflection layer that leads to light guiding along the measuring path. In the fluorescence measurement cell a total internal reflection coating is needed to ensure light guiding of the fluorescence signal. The coating must also be transparent for the excitation light that is coupled into the cell. A new coating material is investigated. The Quicklyzer system is a compact, portable, online measuring tool, that can be used with a $12 \mathrm{~V}$ power supply to take it to the field. It replaces cost and time consuming offline laboratory measuring systems, like inductively coupled plasma mass spectrometry, High-performance liquid chromatography and gas chromatography.
\end{abstract}

Key words: photometric sensor, absorption spectroscopy, fluorescence spectroscopy, total internal reflection, nanoporous material

\section{Introduction}

As public environmental awareness is increasing strongly qualitative and quantitative analysis of organic and ionic contaminations are getting more and more into the center of attention. The continuous measuring of the groundwater provides immense advantages in agricultural and environmental monitoring. On the one hand an accurate monitoring of the metabolism of crops can help to find the requirements of new plant cultivation. On the other hand if the concentration of nitrate is too high, for example through overfertilization, it leads to eutrophication, acidification, changing of flora and fauna and groundwater contamination. So it is necessary to continuously measure the composition of the critical substances in soil water. Also floor loading near landfills and industrial facilities should be observed to detect the escape of harmful substances. [1][2]

Although there are already possibilities to measure continuously, these tools have high flow rates of about $150 \mathrm{ml} / \mathrm{h}$. [3] Soil water only provides very small amounts of sample volume. So for continuous monitoring these tools can't be used. Low detection limits in small sample volumes are at the moment only possible to measure offline in laboratories with huge expensive measuring tools like ICP-MS (inductively coupled plasma mass spectrometry).

Substances of interest in environmental monitoring are nitrate, nitrite and humic acid in the ppm range in aqueous solutions. Different amounts of those substances are present in environmental samples. The measurement of the specific environmental substance is performed in UV/VIS- and fluorescencemeasurement-cells. To be able to measure continuously with small sample volumes the center of competence Nanochem at the University of Applied Sciences in cooperation with Umwelt-Geräte-Technik GmbH (UGT) has developed a small portable device for real-time measurements of ionic and anionic 
contaminations in the ppm range, the Quicklyzer which will be presented here. A special focus will be on new total internal reflection coatings of the cells and the measuring results with the Quicklyzer.

\section{Analysation sensor}

The sensor is a microfluidic liquid core waveguide based on silicon technology. Depending on the application area either a short channel or an up to some meter long spiral measurement channel can be fabricated for measurements from ppm (parts per million) to ppb-range (parts per billion). A patent on the measuring sensor is held by Prof. Lechner and Prof. Hummel. The scientific basis of the spectroscopic measuring method is the interaction of electromagnetic radiation with contaminations in fluids.

Absorption spectroscopy: The measuring principle uses the absorption of electromagnetic radiation. A beam of light which is travelling through the cell is weakened by the absorbent substance. Thereby the extinction $E$ describes the logarithmic quotient of reference intensity $\mathrm{I}_{0}$ and intensity I, influenced by the sample. [3]

$$
E=\lg \frac{I_{0}}{I}
$$

The Beer-Lambert law displays the dependence of the extinction $E$ along a measurement distance I, the concentration $\mathrm{c}$ of the analyte and the molar extinction coefficient $\varepsilon_{\lambda}$. [4]

$$
E=\varepsilon_{\lambda} \cdot l \cdot c
$$

That means that increasing the measurement distance, as it is done with our measuring cell, leads to a decrease of the detection limit. The shorter versions of the cell aim to measure ppm contamination amounts, the longer spiral formed cells are for ppb measurements.

Fluorescence Spectroscopy: In comparison to absorption spectroscopy the emission is shifted to longer wavelengths. The energy difference between the maxima of the absorption and emission spectra is described by the Stokes shift. The following equation is used to calculate the intensity of the fluorescence signal. $\phi$ is the quantum efficiency, $I_{f}$ is the fluorescence intensity, and $\mathrm{I}_{0}$ is the excitation intensity [5]

$$
I_{f}=2,303 \cdot \phi \cdot I_{0} \cdot \varepsilon_{\lambda} \cdot c \cdot l
$$

The measuring cell is based on silicon technology. A newly developed wet etching process is used to etch an isotropic channel in monocrystalline silicon. The channel has a diameter around $300 \mu \mathrm{m}$ with an extremely smooth surface. With the used nanotechnology etching process a channel wall with optical quality (roughness $10 \mathrm{~nm}+/-2 \mathrm{~nm}$ ) is generated. [6] This is important because surface irregulations would lead to enormous losses in light guiding. A release about the isotropic wet chemical etching of deep channels with optical surface quality in silicon will be published.

As silicon absorbs light in the visible range the channel must be coated to generate light guidance. For short measuring channels mirrored reflection cells that are covered with aluminum can be used. A total internal reflective coating leads to even better light guiding along the measuring path. This is achieved if the optical film has a refractive index (n) below the inner core, which will be water with $n=1,33$. Especially for long curved measuring paths of some meters total internal reflection coatings are essential. For fluorescence measurements in the Quicklyzer the excitation light is coupled vertical into the cell. So the coating of the cover must be transparent for the excitation light. Only the fluorescent light is guided through the liquid core waveguide based on the principle of total internal reflection to enhance the yield. The vertical excitation beam is reflected back out of the measuring cell. The only commercial available product which fulfils these requirements is Teflon AF by DuPont ${ }^{\mathrm{TM}}$. Teflon AF was implemented successfully in the fluorescence cells. [6][7][8] But it shows several disadvantages. On the one hand it is very expensive. On the other hand it has low adhesion to most substrates. And there are adhesion problems with glues to fix fluid connectors and glass fibres to the channel. Also it has only a $\Delta n$ to water of about 0.02 . So an alternative material is wanted.

\section{Nanoporous coatings}

A literature research lead to the work with nanoporous materials. The idea is to create a matrix material with air pores. If the pores are sufficient small (at least one magnitude smaller than the used radiation) the matrix material and the pores seem to be a homogeneous film for the light. Than the refractive index is a weighted index of the matrix material and air (refractive index $n=1$ ). The refractive index can be tuned by varying the amount of entrapped air.

As matrix material organosilicat Polymethylsilsesquioxane (PMSSQ) nanoparticles in a colloidal suspension will be used. Crack free films with refractive indizes below $n=1.15$ are for example reported in [9][10][11]. The PMSSQ nanoparticle contains methylgroups which make the coating hydrophobic. The creation of 


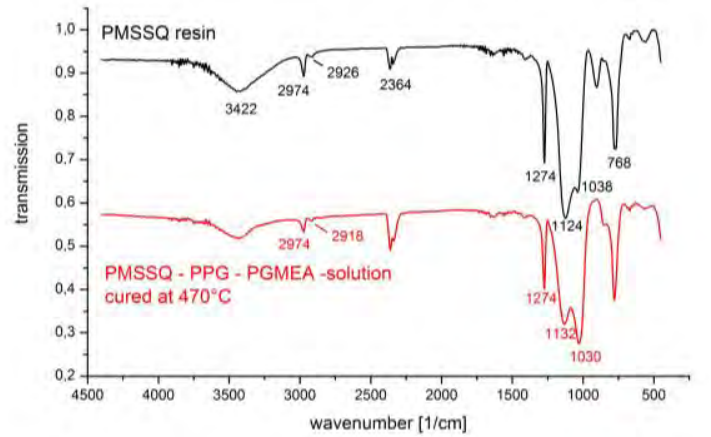

Figure 1 Fourier transform infrared spectroscopy spectra of PMSSQ basic material (top) and of a coating that was cured at $470^{\circ} \mathrm{C}$ (bottom). The coating contained PMSSQ, PPG and PGMEA. Wavenumbers of relevant peaks are marked.

the thin films is done by thermal curing. Pores are built in with a sacrificial porogen approach. The porogen is dissolved in a nanoparticlesolvent-solution and thermally degraded.

The PMSSQ nanoparticles have methyl- $\left(\mathrm{CH}_{3}\right)$, silanol-( $\mathrm{Si}-\mathrm{OH})$ and ether-sidegroups $(\mathrm{R}-\mathrm{O}-$ $\mathrm{C}_{2} \mathrm{H}_{5}$ ). Siloxane-bonds are the backbone of the molecule. Their characteristic is that silicon atoms are directly linked to oxygen atoms $(\mathrm{Si}-$ $\mathrm{O}$ - Si). Water and alcohol condensation reaction of the silanol and the ether groups can take place to build a network of nanoparticles. Polypropylenglycol (PPG) is used as porogen. It is amphilic with an unpolar $\mathrm{CH}_{3}$-group and polar $\mathrm{OH}$-group. Because of this it is good miscible with the nanoparticles. Propylenglycolmonomethylethylacetate PGMEA, is a good solvent for PPG and PMSSQ.

Fig. 1 shows the fourier transform infrared spectroscopy (FTIR) spectrum of the basic material PMSSQ as delivered as flakes and the spectrum of a resulting coating that was cured at $470^{\circ} \mathrm{C}$. The coating contained PMSSQ, PPG and PGMEA and was spin coated onto a silicon substrate. We will concentrate here on the major double peak in the wavenumber region $1000-12001 / \mathrm{cm}$ which is characteristic for siloxane bonds. [10][12] A reversing of the peak heights can be seen, which will be discussed in detail later.

A variation of the curing temperature was done in the temperature region from $245^{\circ} \mathrm{C}$ to $595^{\circ} \mathrm{C}$ in steps of $25^{\circ} \mathrm{C}$. Most samples showed good adhesion on the substrate and homogeneous covering of the substrate. Exceptions were the samples cured above $570^{\circ} \mathrm{C}$ which formed cracks while curing on the hotplate. This temperature series shows that the curing behaviour of the nanoparticles is very strong affected by the curing temperature. Observing the double peak at 1132 and $10301 / \mathrm{cm}$ the peak conversion that was mentioned before can

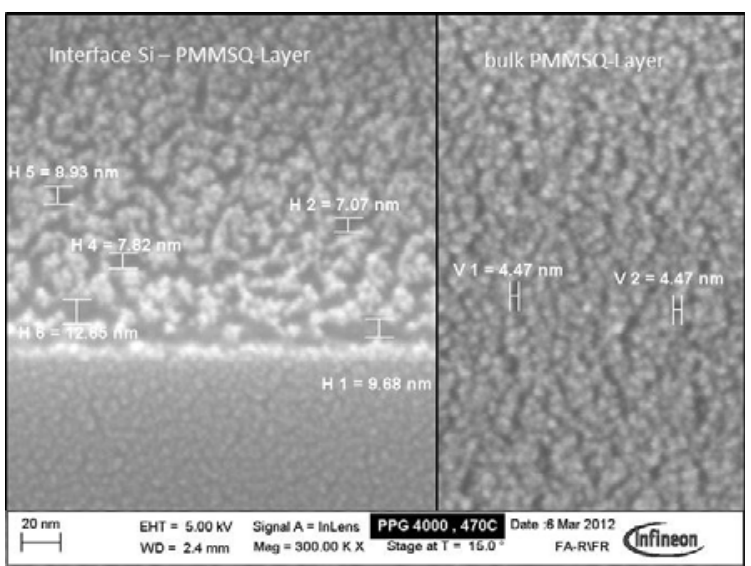

Figure 2 Scanning electron microscope cross section image of a cured PMSSQ film on silicon substrate. Near the substrate is a higher concentration of bigger air pores (left). The average pore diameter near the substrate is $8 \mathrm{~nm}$. About $300 \mathrm{~nm}$ away from the silicon/PMSSQ interface (right) in the center of the coating, the bulk, the average pore diameter is only about $5 \mathrm{~nm}$.

be followed. This is typical for a $\mathrm{Si}-\mathrm{O}-\mathrm{Si}$ backbone with its stretching vibrations. The double peak nature of the PMSSQ material indicates that there are longer and shorter $\mathrm{Si}$ $\mathrm{O}$ - Si chains. The peak at higher wavenumbers stands for stretch bands of $\mathrm{Si}-\mathrm{O}-\mathrm{Si}$ vibrations of laddered structures. This means the material is not so much interconnected. The peak at lower wavenumbers means that there is a higher degree of cross-linking, so it forms a network. Better connection results in higher mass involved in vibration and is therefore lowering the frequency. This conversion is characteristic for the change from a chain to a three dimensional network. [10][12][13] At high temperatures above $495^{\circ} \mathrm{C}$ the characteristic peaks of the methyl-groups are reduced. There is a heat degeneration going on. The material converts to $\mathrm{SiO}_{2}$, to glass. The methylgroups are very important because they make the material hydrophobic which is important for the use as coating for a liquid core waveguide. So the temperature regime must be controlled that way, that the nanoparticles have best connection but still contain the methyl groups. $420^{\circ} \mathrm{C}$ to $495^{\circ} \mathrm{C}$ is a good working regime.

The heating temperature is very important. But also the speed of the heat introduced has significant influence. First SEM investigations showed that there were only minimal pores less than $1 \mathrm{~nm}$ diameter in the coating. They seem to originate only from the interconnection of the nanoparticles. With better heat coupling into the sample higher porosity was generated. SEM investigations showed a decrease of pore loading from bottom of the coating to the top, see Figure 2. As the solution is very viscous 


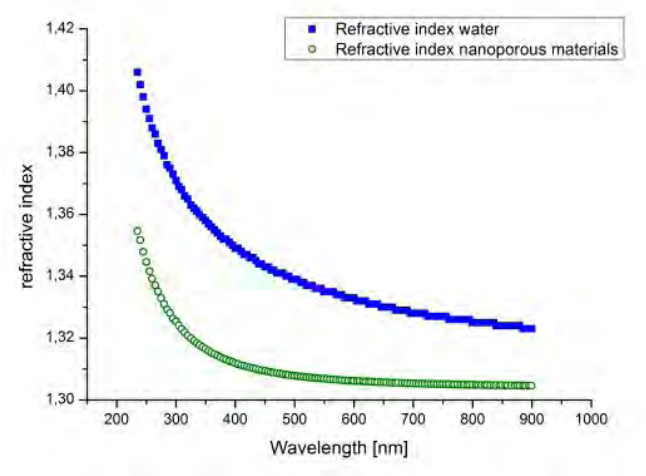

Figure 3 Calculated dispersion curve of the nanoparticle coating (measurement was done with a Filmetrics F20UV white light interferometer) with the spectrum of water.

and the heat enters the solution slowly with a high temperature gradient it seems that the porogen in the bulk will be driven out before the nanoparticle cross linking occurs. So the nanoparticles reflow and close many of the pores. Near the interface the heat seems to arrive suddenly on a very high temperature. Pores with an average pore diameter about $8 \mathrm{~nm}$ are generated. In regions more than $200 \mathrm{~nm}$ away from the interface pores are less and smaller, about $5 \mathrm{~nm}$.

The refractive index of the PMSSQ material is 1.42 as pointed out by the company Techneglas. In Figure 3 two dispersion curves of the material and water in the wavelength region 380nm - 900 $\mathrm{nm}$ are displayed. The measurements show a reduction of the refractive index of the new coating below the one of water. On test substrates good results were achieved. So in the next step we implement the new coating in the measuring channel to replace Teflon AF.

\section{Measuring system}

The experimental setup for absorptiometry and fluorescence measurement is shown in Figure 4. Before measuring the sample in the cell the fluid is filtrated and all air bubbles are separated. A UV/VIS-light source stimulates the molecules in absorption measurements. The lowered radiation caused by self-absorption is detected by a spectrometer at the end of the measuring cell. A special software, which has been developed [14] for this measurement setup calculates the amount of the substances in the liquid sample with the usage of calibration lines. The second measurement setup is fluorescence measurement. The difference in the measurement setup is the excitation method. Here a high-power UV-LED is used to generate emission. The light is coupled vertical into the cell instead of axial radiation. This

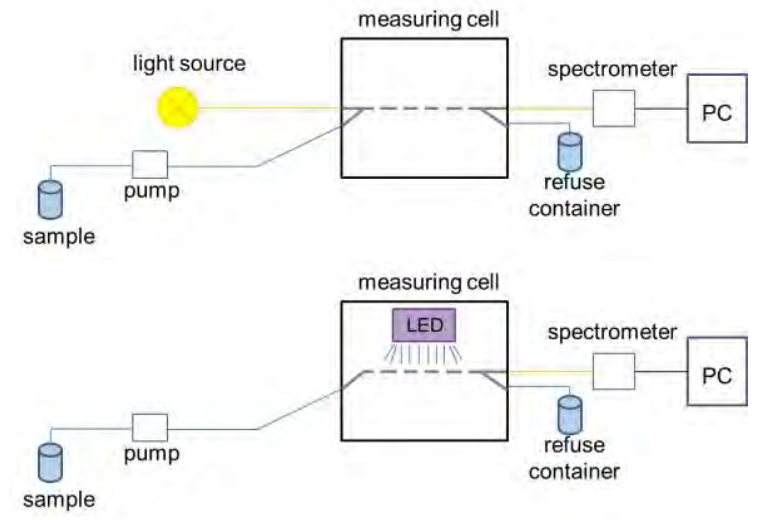

Figure 4: Schematic picture of the experimental setup for absorption (top) and florescence (bottom) measurements. [15]

setup enables a measurement without blocking filter because only the fluorescent light is guided through the liquid core waveguide based on the principle of total internal reflection. The vertical excitation beam is reflected back out of the measuring cell.

With regard to environmental monitoring the photometric sensor was integrated in an environmental measuring system called Quicklyzer in cooperation with the company Umwelt-Geräte-Technik $\mathrm{GmbH}$. In the environmental sector the requirements are in the parts per million sector (ppm). The amount of nitrate, nitrite and humic acid can be measured by the combination of absorption and fluorescence measurements.

The Quicklyzer can work with smallest amounts of sample volume in continuous measuring mode below $2,5 \mathrm{ml} / \mathrm{h}$. Special is the small size, the wireless data transfer via GSM and a $12 \mathrm{~V}$ supply. This makes an automated onlinemeasurement in the field possible. Only small operator costs are necessary. With this system dynamic processes of degradation or conversion during the transport to the laboratories can be suppressed.

The Quicklyzer is outstanding because it is possible to measure nitrate, nitrite and humic acid in one single device. So it replaces the measurements with expensive offline tools. Devices like inductively coupled plasma mass spectrometry (ICP-MS), high-performance liquid chromatography (HPLC) and gas chromatography (GC) would be used instead. You also avoid transportation of the sample to the lab.

A second project of photometric water analysis is done with regard to semiconductor industries at the University of Applied Sciences Regensburg. In the project Picolyzer a measurement system for online surveillance of 


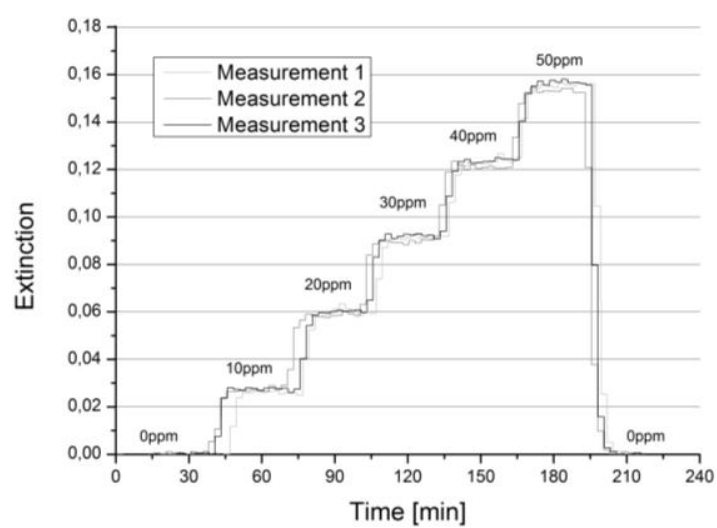

Figure 5: Time response of the extinction as function of the nitrite concentration. The measurements are done in a mirrored reflection cell. [15]

process-wet-chambers in the semiconductor industry is developed. The resolution of the system will be in the sub-ppb area (parts-perbillion, $10^{-9}$ ). Currently there is no possibility for real-time controls while cleaning processes. [6]

\section{Measurements}

With the Quicklyzer you can measure nitrate and nitrite via self-absorption. Figure 5 shows the time response of the extinction signal as function of the nitrite calibration reference solutions. It shows that the absorption value is constant until the sample is changed. The measurement has been done three times with the same solutions to display that the measurement is reproducible. The deviation of about $\pm 1.6 \%$ is within tolerance. The results from this measurement can be used to calibrate the software so that it is possible to measure different concentrations. The fitted calibration line shows that the regression line approximates $99.9 \%$.

Using a UV-LED makes it possible to dislocate the absorption band of humic acid from the UV region to the visible region. With the special wavelength of the LED it is also possible to distinguish between humic acids and other organic substances. Because of the stokes shift a fluorescent maximum can be observed. First a spectrum with a reference solution (deionized water) was taken. After that different samples of humic acid with known concentrations from 10 to $50 \mathrm{ppm}$ were analyzed.

Figure 6 shows the results of the measurement. As the experiments were done three times it is obvious that the measurements have a very good reproducibility. A disadvantage of fluorescence spectroscopy is that if the maximum concentration $c_{\max }$ is reached the fluorescence intensity will deepen. That means that the direct proportionality between concentration and fluorescence intensity is not

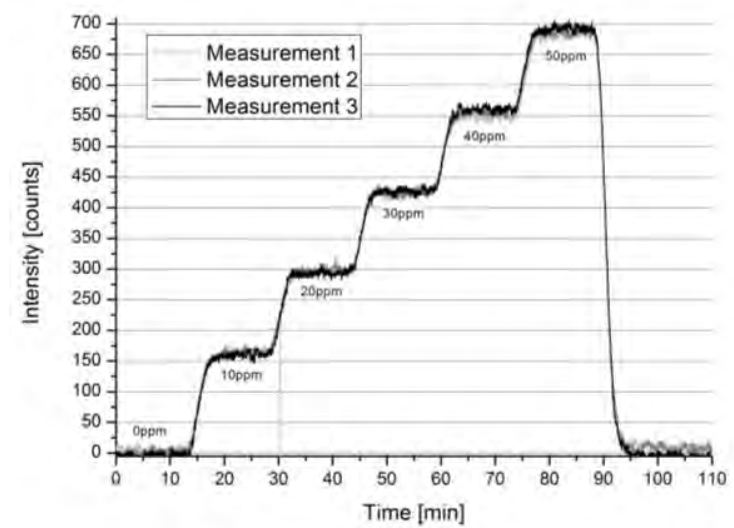

Figure 6 Fluorescence measurement of different concentrations of humic acid. The measurements are done in a florescence cell with a Teflon AF total internal reflection coating.

valid any more. That is known as fluorescence quenching. Consequential it is only possible to measure humic acid concentrations until a certain upper limit. The measurement system should also be prevented from temperature drifts because this would lead to lattice vibrations of the molecules and therefore the fluorescence intensity would be influenced. [1]

To evaluate the Quicklyzer a test of the nitrate concentrations in soil water from a field of the company UGT in Müncheberg, Germany has been done. The measurements were done within eight days with the percolate water of a lysimeter. Two samples of the percolate water were taken on each day, always at the same time of the day. They were measured by the Quicklyzer and also sent to two different certified laboratories. Figure 7 shows the comparison of the measurements with the Quicklyzer and the results of the laboratories. The Leibniz Centre for Agricultural Landscape Research used ion chromatography and the State Control Association Brandenburg used a photometric measurement system like the Quicklyzer. The Quicklyzer shows comparability with these certified laboratories.

\section{Résumé}

A new online measurement system with low sample volume for environmental relevant substances is realized with the Quicklyzer. The centerpiece of the measuring system is the measuring cell. Dependent on the cell design different analysis limits can be realized from ppm to ppb region. This analytical system has even more potential in terms of detecting different substances which will be explored in the further work.

It was shown that new optical nanoporous coatings with a refractive index can be generated. Insights in the curing behavior were 


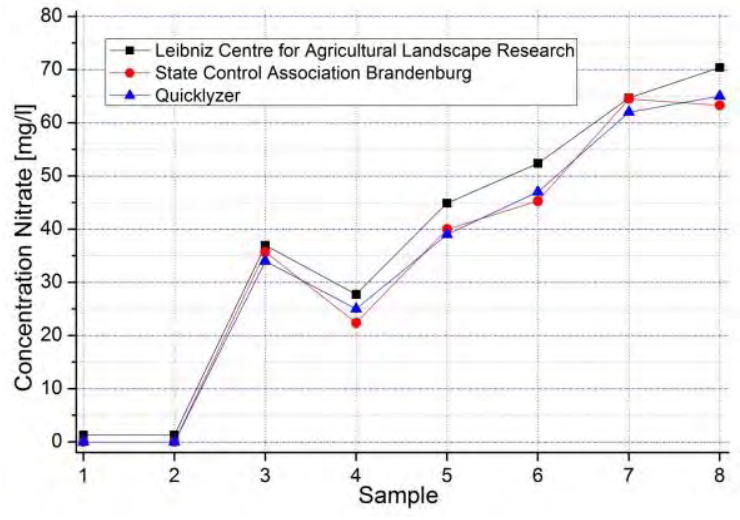

Figure 7 Comparison of the detected nitrate concentration of two different laboratories and the Quicklyzer. The measurements are done in a mirrored reflection measuring cell.[16]

gained. With a good temperature coupling a three dimensional porous network can be achieved.

As the measurement of nitrate and nitrite is done only via self-absorption, it is not necessary to add other substances for analysis which makes the measurement extremely easy. Measuring of humic acid via fluorescence spectroscopy with a special UV-LED makes it possible to differ this organic substance from other organic substances. A field test showed clearly that the measurement can be compared with the measurements of different certified institutes.

\section{Acknowledgement}

Special thanks to ZIM (Zentrales Innovationsprogramm Mittelstand; Support code: ZIM KF2185302KMO) for their financial support. We also thank the project partner UGT for the good collaboration and the staff of the centers of competence Nanochem and Sensorik.

\section{References}

[1] [Online] J. Block, „Stickstoffsättigung“, http://www.wald-rlp.de/fileadmin/website/ fawfseiten/fawf/FUM/umweltmonitoring/stickstoffs aettigung.html.[6. March 2012].

[2] F. Lechner, Floureszenz und Absorption von organischen Verbindungen, Hochschule Regensburg, 2011

[3] M. de Rechter, Skalar analytic $\mathrm{GmbH}$, Analytica 2012, Munich, Germany, April 2012

[4] T. Owen, Fundamentals of UV-visible spectroscopy, Germany: Agilent Technologies 2000, 2000, Publication number 5980-1397E

[5] J.R. Albani, Principles and Application of Flourescence Spectroscopy, Blackwell Publishing, 2007, ISBN: 978-1-4051-3891-8
[6] A. Hutterer, M. Bauhuber, M. Argauer, A. Mikrievskij, H. Hummel, A. Lechner, Analysesystem zur Online-Messung von Kleinstkontaminationen im ppb-Bereich, Tagungsband 3. Landshuter Symposium Mikrosystemtechnik 2012, 295-302 (Landshut 2012), ISBN: 978-3-9812696-2-8

[7] M. Zitzelsberger, M. Argauer, M. Bauhuber, M. Betz, A. Graf, A. Hutterer, A, Mikrievskij, $H$. Hummel, A. Lechner, Evaluation and Water Resistance of Different Adhesion Promoters for Teflon $\mathrm{AF}^{\circledR}$ Layers for Quicklyzer Measuring cell, Applied Research Conference 2012, 154-159 (Nürnberg 2012), ISBN: 978-3-8440-1093-0

[8] M. Betz, A. Graf, M. Zitzelsberger, F. Lechner, H. Hummel, A. Lechner, Quicklyzer Photometrisches Messgerät zur Spurenanalyse von Bodenstoffen im ppm-Bereich, Tagungsband 3. Landshuter Symposium Mikrosystemtechnik 2012, 235-243 (Landshut 2012), ISBN: 978-39812696-2-8

[9] W. Risk, H. Kim, R. Miller, H. Temkin, S. Gangopadhyay, Optical waveguides with an aqueous core and a low-index nanoporous cladding, Opt. Express, Vol. 12, Issue 26, 64466455 (2004), DOI: 10.1364/OPEX.12.006446

[10] H.-C. Kim, J.B. Wilds, C.R. Kreller, W. Volksen, P.J. Brock, V.Y. Lee, T. Magbitang, J.L. Hedrick, C.J. Hawker, R.D. Miller, Fabrication of Multilayered Nanoporous Poly(methylsilsesquioxane), Advanced Materials, Vol.14, No. 22, 1637-1639 (2002), DOI: 10.1002/1521-4095(20021118)

[11] V. Korampally, M. Yun. T. Rajagopalan, P. K Dasgupta, K. Gangopadhyay, S. Gangopadhyay, Entropy driven spontaneous formation of highly porous films from polymernanoparticle composites, Nanotechnology 20, 42, 2009 doi:10.1088/0957-4484/20/42/425602

[12] A. M. Padovani, L. Rhodes, S. A. Bidstrup Allen, P. Kohl, Chemically Bonded Porogens in Methylsilsesquioxane, Journal of The Electrochemical Society 149 (12), 161-170 (2002), DOI: 10.1149/1.1515281

[13] R. H. Baney, M. Itoh, A..Sakakibara, T. Suzuki, Silsesquioxanes, Chemical Reviews 95, 5, 14091430(1995), DOI: 10.1021/cr00037a012

[14] Bauer, Andreas, MEDUSA Software, Regensburg 2008.

[15] A.Graf, M. Bauhuber, A. Hutterer, F. Lechner, M. Argauer, M. Zitzelsberger, A. Lechner, $\mathrm{H}$. Hummel, Quicklyzer - A new Developed Analytical Measurement System for Online Measurements of Specific environmental Substances, Applied Research Conference 2012, 147-150 (Nürnberg 2012), ISBN: 978-3-84401093-0

[16] Umwelt-Geräte-Technik $\mathrm{GmbH}$, Müncheberg 2011 